\title{
Spillovers de exportación y supervivencia exportadora en España*
}

\section{Export Spillover and Export Survival in Spain}

\author{
Silviano Esteve \\ Francisco Requena \\ Universitat de València \\ Juan de Lucio \\ Universidad de Alcalá de Henares \\ Raul Mínguez \\ Cámara de Comercio de España \\ Universidad de Nebrija \\ Asier Minondo \\ Universidad de Deusto
}

\section{Resumen}

Utilizando datos de empresas manufactureras exportadoras españolas, este trabajo investiga si la presencia de empresas que exportan de forma regular un producto a un país ayuda a otras empresas en la misma provincia que empiezan a exportar a mantener relaciones de exportación más duraderas. Nuestros resultados muestran que: (i) la tasa de supervivencia de las nuevas empresas exportadoras es mayor en las relaciones de exportación definidas a nivel de provincia-producto-destino cuando hay presencia de al menos un exportador regular en la misma provincia; (ii) cuando hay más de 10 exportadores regulares en la misma provincia, el impacto positivo se incrementa significativamente; y (iii) el efecto positivo de la concentración de exportadores regulares es específico al nivel provincia-producto-destino puesto que el impacto es menor o no existe a nivel provincia-producto, provincia-industria o provincia-destino.

Palabras clave: externalidades, actividad exportadora, empresas regulares, nuevos exportadores, modelos de duración.

Clasificación JEL: F14.

\begin{abstract}
Using data from Spanish exporting manufacturing firms, this paper investigates whether the presence of firms that regularly export a product to a country helps other nearby companies that start exporting to maintain longer-lasting export relationships. First, we find clear evidence that

* Los autores agradecen al Departamento de Aduanas e Impuestos Especiales de la Agencia Tributaria por el acceso a los datos de exportación. Agradecemos la financiación recibida por el Ministerio de Economía, Industria y Competitividad (RTI2018-100899-B-I00, cofinanciado con FEDER), del Departamento de Educación, Política Lingüística y Cultura del Gobierno Vasco (IT885-16), de la UAH - Santander Universidades (2019/00003/016/001/007) y de la Generalitat Valenciana (GVPrometeo 2018/102).
\end{abstract}


the survival rate of new exporting companies is higher in those export relationships defined at the province-product-destination level when there is at least one regular exporter. Second, when there are more than 10 regular exporters in the same province, the positive impact increases significantly. Third, the positive effect of the concentration of regular exporters is very specific: the impact is smaller or null at the level of province-product, province-industry or province-destination.

Keywords: externalities, survival, new exporters, duration models.

JEL Classification: F14.

\section{Introducción}

Exportar es difícil. La literatura existente enfatiza que comenzar a exportar es costoso y que además las relaciones de exportación suelen tener una corta duración, esto es, la supervivencia es complicada. A menudo exportar implica tener que incurrir en una inversión no recuperable antes de poder empezar a vender fuera: investigar y conocer los mercados extranjeros, establecer redes de distribución o adaptar los productos a los gustos de los consumidores extranjeros. Dado que estos costes de entrada son intensivos en conocimiento, los exportadores con experiencia pueden tener un impacto positivo en el desempeño de otras empresas que empiezan a exportar. En la literatura de comercio internacional, los beneficios que unos exportadores aportan a otras empresas cercanas se conoce como «spillovers de exportación» (nos referiremos en el texto como $S E$ ). Dado que los $S E$ tienden a estar altamente localizados y ser específicos a un producto y/o destino, es más probable que los beneficios de empezar a exportar a nivel de empresa-producto-destino aumenten cuando hay una elevada concentración geográfica de empresas vendiendo el mismo producto al mismo destino. En otras palabras, la aglomeración de exportadores puede incitar a otras empresas cercanas a exportar los mismos productos a los mismos países y a facilitar que estas nuevas relaciones de exportación perduren.

Hay varios argumentos que explican por qué la concentración geográfica de exportadores de una misma actividad exportadora influye positivamente en el desempeño exportador de empresas cercanas. Con respecto a la decisión de empezar a exportar, Krautheim (2012) argumenta que el intercambio de información entre las empresas que exportan a un mismo destino reduce los costes fijos de exportación a nivel de empresa y, por tanto, aumenta la propensión exportadora a nivel local. Con respecto al margen intensivo de exportaciones, Rauch y Watson (2003) defienden que la presencia de exportadores con experiencia reduce la incertidumbre de los compradores extranjeros en términos de la calidad de los proveedores de la misma región; esto típicamente favorece pedidos más grandes y, por tanto, más exportaciones. Alternativamente, Cassey y Schmeiser (2013) enfatizan que un número elevado de exportadores locales reduce los costes variables de comercio, por ejemplo, compartiendo contenedores. Así pues, la aglomeración geográfica de exportadores puede actuar como facilitador del comercio a través de la reducción de los costes fijos y variables de exportación. 
Este trabajo investiga el papel de los $S E$ en el desempeño exportador de las empresas manufactureras españolas que empiezan a exportar. Más específicamente, examinamos el impacto de la aglomeración geográfica de exportadores con experiencia (que llamaremos exportadores regulares) sobre la duración de las nuevas relaciones de exportación de empresas que nunca antes habían exportado (que llamaremos nuevos exportadores).

Varios estudios han explorado la existencia de $S E$, con conclusiones dispares. Recientemente, Duan et al. (2020) realiza un meta-análisis de los $S E$ centrado en la relación entre la aglomeración geográfica de empresas y las decisiones de exportar o no exportar, y dónde exportar ${ }^{1}$. Hay tres resultados principales. Primero, la cobertura de países analizados es muy amplia y la gran mayoría obtiene evidencia a favor de que la concentración espacial de exportadores mejora el desempeño exportador de otras empresas cercanas. Segundo, el diseño del análisis empírico no permite establecer una relación causal en la mayoría de casos y los resultados en general no son comparables. Tercero, cuando los resultados son comparables, el meta-análisis concluye que hay $S E$, pero su impacto es económicamente muy pequeño.

Para España, la evidencia es todavía escasa y mixta. Con datos a nivel de empresa, Barrios et al. (2003) y Máñez et al. (2004) no encuentran evidencia a favor de $S E$ en la decisión de empezar a exportar entre las empresas manufactureras usando la base de datos «Encuesta sobre Estrategias Empresariales» (ESEE). Con datos a nivel de empresa-destino, Requena y Castillo (2007) y Muñoz y Rodríguez (2015) encuentran evidencia a favor de $S E$ a nivel geográfico en la decisión de adónde exportar con dos muestras diferentes de empresas manufactureras. Con datos a nivel de empresa-producto-destino, de Lucio et al. (2020) encuentra evidencia a favor de $S E$ en la decisión de iniciar una nueva relación de exportación usando el universo de empresas exportadoras entre 1998 y 2018. Además, también observa que los $S E$ son mayores cuando las empresas que empiezan a exportar no tienen experiencia previa (no exportaron el año anterior).

A diferencia de trabajos previos para España, este trabajo investiga los $S E$ originados por los exportadores regulares (con experiencia al menos de 4 años exportando) sobre los nuevos exportadores (sin ninguna experiencia exportadora previa) y utiliza como medida de desempeño exportador la duración de las nuevas relaciones de exportación definidas a nivel de empresa-producto-destino (EPD) ${ }^{2}$.

El principal resultado del trabajo es que la probabilidad de que una nueva relación de exportación de una empresa sin experiencia exportadora previa sobreviva más allá de su primer año aumenta más de un $50 \%$ cuando hay al menos otra empresa manufacturera con experiencia en la provincia vendiendo ese producto en ese destino. Cuando controlamos por características de empresa, industria, producto y

\footnotetext{
${ }^{1}$ En su revisión de la literatura, ponen de manifiesto la abundancia de trabajos que analizan si la presencia cercana de una multinacional extranjera ayuda a las empresas locales en su desempeño exportador. Sin embargo, los trabajos que examinan estos $S E$ no son incluidos en el meta-análisis.

2 Nuestro trabajo adopta la misma estrategia que Requena y Castillo (2007) en su análisis de la decisión de adónde exportar al seleccionar solamente empresas sin experiencia exportadora previa.
} 
destino, la probabilidad de supervivencia en el primer año aumenta en torno al $75 \%$ a partir de una aglomeración de 10 exportadores regulares en una provincia.

Los trabajos más parecidos al nuestro son Esteve et al. (2013) y Esteve et al. (2017). Sin distinguir el tipo de actividad que realiza la nueva empresa o los años de experiencia exportadora de las empresas locales, los dos trabajos encuentran que la aglomeración de empresas con una misma actividad de exportación tiene un impacto positivo, pero muy pequeño (y a veces nulo), sobre la duración de las relaciones de exportación a nivel de empresa-destino (2000-2010) y de empresa-producto-destino (2000-2015). Hay dos posibles explicaciones (Ahn et al., 2011; Choquette, 2019). La primera es que el aprendizaje basado en una experiencia exportadora previa reduce significativamente el papel de los $S E$ en la probabilidad de supervivencia de las relaciones de exportación. La segunda es que los costes hundidos asociados a comenzar a exportar son diferentes según el tipo de actividad de la empresa; por ejemplo, las empresas comercializadoras incurren en menores costes de entrada en cada mercado que las empresas manufactureras, aumentando la frecuencia de las transacciones puntuales y reduciendo el papel de los $S E$. En nuestro trabajo, el papel de los $S E$ es importante entre las empresas manufactureras que no tienen experiencia exportadora previa.

El resto del trabajo se organiza de la siguiente manera. La sección 2 presenta los datos, las variables y algunas estadísticas descriptivas relacionadas con las características de los nuevos exportadores. La sección 3 presenta el modelo empírico. La sección 4 presenta los resultados empíricos y varios ejercicios de robustez. La sección 5 finaliza con las conclusiones.

\section{Datos}

La actividad exportadora se puede medir combinando características de empresa, industria, origen, producto y destino. Cuando hablamos de una relación de exportación, nos referimos a una empresa que exporta un producto a un país, de modo que nuestra unidad de observación es el triplete «EPD» o empresa-producto-destino. Además, hay dos dimensiones adicionales a tener en cuenta: la actividad manufacturera principal que realiza la empresa (industria) y la provincia en la que tiene la sede social la empresa (origen). En este trabajo utilizamos el micro-dato de la base de datos de AEAT-Aduanas para el que hemos tenido acceso a dos tipos de empresas manufactureras cuyo valor de exportación anual superaba los 6.000 euros en 2004: (1) exportadores nuevos -empresas que empezaron a exportar en 2004 sin haberlo hecho en los tres años previos; (2) exportadores regulares -empresas que exportaron cuatro años consecutivamente hasta 2004. Para todas las empresas conocemos el valor FOB de cada transacción de exportación e importación a nivel de producto (a cuatro dígitos según la nomenclatura del sistema armonizado -partidas) y a nivel de destino (país) en 2004, así como la provincia en la que la empresa tiene su establecimiento exportador. Para los exportadores nuevos también conocemos el número de años que ha exportado cada transacción desde 2004 hasta 2018. 
Para caracterizar las aglomeraciones utilizamos tres dimensiones: provincia de origen de la empresa, producto y destino. Por tanto, una aglomeración se define como el número de exportadores regulares en el triplete provincia-producto-destino en 2004. Combinando dimensiones podemos medir otras formas de aglomeración dentro de la provincia: exportadores regulares del mismo producto a otros países y exportadores regulares de productos diferentes al mismo destino. La idea básica detrás de esta variable es que cuanto mayor sea la aglomeración, es decir, el número de exportadores regulares realizando la misma actividad de exportación, mayor será la influencia sobre otras empresas, ya sea por transmisión de información, costes compartidos, cooperación en desarrollos tecnológicos, etc.

Al utilizar información de exportadores nuevos y regulares resolvemos los principales problemas que tienen la mayoría de trabajos en la literatura de $S E$. Primero, como todas las empresas son nuevas exportadoras no hay que preocuparse por controlar la experiencia exportadora previa. Segundo, en la medición de la existencia y tamaño de la aglomeración solo se cuentan las empresas regulares exportadoras, lo que elimina el problema de endogeneidad entre la decisión de entrada y salida de otras empresas antes de 2004 y la formación de aglomeraciones a nivel de producto-destino. Tercero, los exportadores regulares tienen al menos tres años de experiencia previa acumulada, luego permitimos que los $S E$ surjan en años anteriores a la decisión de empezar a exportar.

\subsection{Nuevas empresas exportadoras}

El Cuadro 1 presenta algunos descriptivos básicos de las empresas exportadoras utilizadas en el trabajo. Según la base de datos AEAT-Aduanas, había 8.370 nuevas empresas exportadoras en 2004 (manufactureras o no) que establecieron al menos una nueva relación de exportación por valor superior a 6.000 euros. Estas empresas exportaron 952 productos a 158 países. La base de datos Cámaras-Directorio de empresas manufactureras (DIRMAN) contiene 1.238 empresas, vendiendo 555 productos a 114 países diferentes ${ }^{3}$. Esta muestra de empresas acumula el $34 \%$ del valor de exportación de los nuevos exportadores en 2004. Para el análisis empírico hemos seleccionado solamente las relaciones de exportación EPD de las nuevas empresas exportadoras que representan más del $1 \%$ de las ventas de exportación de la empresa en 2004. El número de EPD de estas empresas es 2.740, exportando 536 productos a 108 países.

El Cuadro 2 muestra la distribución de empresas según el número de pares producto-destino (EPD). El principal EPD de cada empresa acumula casi el $58 \%$ del valor de exportación inicial de los nuevos exportadores. Dos tercios de las nuevas empresas exportan un único producto a un solo mercado. A medida que aumenta la

${ }^{3}$ Se han excluido destinos que no son países (por ejemplo, Avituallamiento) y países con menos de 100.000 habitantes (por ejemplo, Gibraltar y Andorra). 


\section{CUADRO 1 \\ DESCRIPCIÓN DE LA BASE DE DATOS}

\begin{tabular}{|l|c|c|c|c|c|}
\hline & $\begin{array}{c}\text { Número de } \\
\text { empresas }\end{array}$ & $\begin{array}{c}\text { Número de } \\
\text { productos }\end{array}$ & $\begin{array}{c}\text { Número de } \\
\text { países }\end{array}$ & $\begin{array}{c}\text { Número de } \\
\text { relaciones }\end{array}$ & $\begin{array}{c}\text { \% valor de } \\
\text { las expor- } \\
\text { taciones }\end{array}$ \\
\hline Nuevos exportadores & 8.370 & 952 & 158 & 18.236 & 100 \\
\hline AEAT Aduanas & 3.321 & 795 & 135 & 8.312 & 76 \\
\hline Camaras Directorio & 1.238 & 555 & 114 & 3.306 & 34 \\
\hline Direc. Manuf. (DIRMAN) & 1.238 & 536 & 108 & 2.740 & 33 \\
\hline EPD>1\% exportaciones totales & & & & \\
\hline Exportadores regulares & 23.509 & 1.213 & 174 & 325.737 & 100 \\
\hline AEAT Aduanas & 19.240 & 1.211 & 174 & 313.733 & 98 \\
\hline Camaras Directorio & 11.969 & 1.175 & 174 & 203.268 & 73 \\
\hline Direct. Manuf. (DIRMAN)
\end{tabular}

NOTAS:

- Una relación de exportación «EPD» viene dada por tres dimensiones: empresa (o su localización), producto y destino.

- Se incluyen solo las relaciones de exportación por valor superior a 6.000 euros y cuyo origen es la Península Ibérica.

- Se han excluido los capítulos 97, 98 y 99 del Sistema Armonizado. Productos definidos a 4 dígitos del Sistema Armonizado.

- Se han excluido destinos que no son países (por ejemplo, Avituallamiento) y países con menos de 100.000 habitantes (por ejemplo, Gibraltar y Andorra).

- Una empresa es nueva exportadora cuando empieza a exportar y nunca ha exportado antes en el periodo 1997-2003.

- Una empresa es exportadora regular cuando ha exportado todos los años entre 2001 y 2004.

- Aduanas y Directorio incluyen todas las empresas exportadoras con independencia de su actividad principal.

- Nuestra muestra DIRMAN incluye solo empresas cuya actividad principal es manufacturas (CNAE 1000-3299).

diversificación, el número de nuevas empresas se reduce, pero su peso en el valor total de las nuevas exportaciones aumenta. Solo hay 32 nuevos exportadores con más de 11 combinaciones producto-destino, un $3 \%$ del total, pero acumulan casi el $30 \%$ del total del valor de las nuevas exportaciones.

Cada relación de exportación EPD la realiza una empresa tomando de su cartera un producto y un destino. El Cuadro 3 complementa la información del cuadro anterior: tres cuartas partes de las nuevas empresas exportadoras solo vende un producto manufacturado o solo vende a un país. Entre las nuevas empresas multiproducto-multidestino, el mayor peso en el valor de exportaciones la tienen las empresas que venden dos o tres productos a más de tres destinos. Este resultado contrasta con el observado para el conjunto de las empresas exportadoras españolas, donde unas pocas empresas regulares multiproducto-multidestino acumulan la mayoría de las exportaciones. 


\section{CUADRO 2}

DISTRIBUCIÓN DE NUEVAS EMPRESAS SEGÚN EL NÚMERO DE PARES PRODUCTO-DESTINO (EPD)

\begin{tabular}{|l|c|c|c|}
\hline & Número & \% de empresas & $\begin{array}{c}\text { \% valor de las } \\
\text { exportaciones }\end{array}$ \\
\hline Número de EPD & 2.740 & 100 & 100 \\
\hline Top EPD de cada empresa & 1.238 & 100 & 57,9 \\
\hline Empresas con 1 EPD & 811 & 65,5 & 13,1 \\
\hline Empresas con 2-4 EPD & 294 & 23,7 & 22,2 \\
\hline Empresas con 5-10 EPD & 101 & 8,2 & 35,1 \\
\hline Empresas con +11 EPD & 32 & 2,6 & 29,6 \\
\hline
\end{tabular}

NOTA: Una relación de exportación «EPD» viene dada por tres dimensiones: empresa, producto y destino. FUENTE: Elaboración propia con datos de Cámaras-DIRMAN.

\section{CUADRO 3}

\section{DISTRIBUCIÓN DEL NÚMERO DE NUEVOS EXPORTADORES Y VALOR DE EXPORTACIÓN (EN PARÉNTESIS) POR NÚMERO DE DESTINOS Y PRODUCTOS EN 2004}

\begin{tabular}{|c|c|c|c|c|}
\hline \multirow{2}{*}{$\begin{array}{c}\text { Número de } \\
\text { productos } \\
\text { (HS 4 dígitos) }\end{array}$} & \multicolumn{3}{|c|}{ Número de destinos } & \multirow{2}{*}{ Total } \\
\cline { 2 - 5 } & $\mathbf{1}$ & De 2 a 3 & $\mathbf{+ 3}$ & \\
\hline 1 & 65,7 & 7,9 & 4,2 & 77,8 \\
& $(13,1)$ & $(9,6)$ & $(17,7)$ & $(40,4)$ \\
\hline De 2 a 3 & 6,4 & 6,8 & 4,2 & 17,4 \\
& $(2,3)$ & $(15,4)$ & $(28,3)$ & $(46,0)$ \\
\hline+3 & 1,7 & 1,4 & 1,7 & 4,8 \\
& $(5,0)$ & $(2,4)$ & $(6,1)$ & $(13,5)$ \\
\hline Total & 73,8 & 16,1 & 10,1 & 100 \\
& $(20,4)$ & $(27,4)$ & $(52,1)$ & $(100)$ \\
\hline
\end{tabular}

NOTA: El número de empresas exportadoras nuevas es 1.238.

FUENTE: Elaboración propia con datos de Cámaras-DIRMAN.

Para explorar algunos patrones generales de la actividad exportadora de las nuevas empresas manufactureras exportadoras en España, el Cuadro 4 presenta la distribución de las 1.238 nuevas relaciones de exportación empresa-producto-destino más importantes (top EPD) por destino y por tipo de producto (definido a nivel de secciones del Sistema Armonizado). Claramente los principales socios comerciales de los nuevos exportadores son nuestros vecinos (Francia y Portugal con un $11 \%$ y $10 \%$ ), seguido de EEUU (9\%), Marruecos (7\%) y otros destinos europeos (Italia, Alemania, Suiza y Reino Unido). El top 10 representa el $58 \%$ del número total de nuevas relaciones comerciales. 


\section{CUADRO 4}

\section{DISTRIBUCIÓN DE LAS RELACIONES DE EXPORTACIÓN PRINCIPALES (TOP EDP) DE LOS NUEVOS EXPORTADORES EN 2004}

\begin{tabular}{|c|c|c|c|l|c|c|c|}
\hline $\begin{array}{c}\text { Destinos } \\
\text { (top EPD) }\end{array}$ & País & Empresas & $\begin{array}{c}\text { \% } \\
\text { empresas }\end{array}$ & $\begin{array}{c}\text { Productos } \\
\text { (Top EPD) }\end{array}$ & $\begin{array}{c}\text { HS } \\
\text { 2 dígitos }\end{array}$ & Empresas & $\begin{array}{c}\text { \% } \\
\text { empresas }\end{array}$ \\
\hline Top 1 & Portugal & 142 & 11 & $\begin{array}{l}\text { Alimentos, } \\
\text { bebida }\end{array}$ & $00-24$ & 187 & 15,1 \\
\hline Top 2 & Francia & 123 & 10 & Minerales & $25-27$ & 19 & 1,5 \\
\hline Top 3 & EE UU & 112 & 9 & Química & $28-38$ & 67 & 5,4 \\
\hline Top 4 & Marruecos & 83 & 7 & $\begin{array}{l}\text { Plástico, } \\
\text { caucho }\end{array}$ & $39-40$ & 90 & 7,3 \\
\hline Top 5 & Italia & 72 & 6 & $\begin{array}{l}\text { Piel, cuero, } \\
\text { madera, papel }\end{array}$ & $41-49$ & 115 & 9,3 \\
\hline Top 6 & Alemania & 49 & 4 & $\begin{array}{l}\text { Textil, } \\
\text { confección } \\
\text { y calzado }\end{array}$ & $50-67$ & 213 & 17,2 \\
\hline Top 7 & Mexico & 47 & 4 & $\begin{array}{l}\text { Minerales no } \\
\text { metálicos }\end{array}$ & $68-70$ & 49 & 3,9 \\
\hline Top 8 & Suiza & 42 & 3 & Metales & $72-83$ & 117 & 9,5 \\
\hline Top 9 & Reino Unido & 33 & 3 & $\begin{array}{l}\text { Maq. mecáni- } \\
\text { ca y eléctrica }\end{array}$ & $84-85$ & 211 & 17,0 \\
\hline Top 10 & Turquía & 21 & 2 & $\begin{array}{l}\text { Equipo } \\
\text { transporte }\end{array}$ & $86-89$ & 54 & 4,4 \\
\hline $\begin{array}{c}\text { Resto } \\
\text { destinos }\end{array}$ & 98 países & 514 & 42 & $\begin{array}{l}\text { Manufacturas } \\
\text { diversas }\end{array}$ & $71,90-96$ & 116 & 9,4 \\
\hline
\end{tabular}

FUENTE: Elaboración propia con datos de Cámaras-DIRMAN.

Por otro lado, se observa que las nuevas relaciones de exportación según el producto manufacturado más frecuentes son en textil, confección y calzado (17\%), maquinaria mecánica y eléctrica (17\%) e industria agroalimentaria (15\%). Le siguen los productos metálicos (9\%), la manufactura de la piel, cuero, madera, papel (9\%) y la manufactura del mueble, juguete y joyería $(9 \%)$.

\subsection{Aglomeración de exportadores regulares}

Volviendo al Cuadro 1, según los datos de AEAT-Aduanas, en 2004 había 23.509 empresas exportadoras regulares (manufactureras o no) vendiendo 1.213 productos (definido a 4 dígitos del Sistema Armonizado) a 174 países. La base de datos Cámaras-Directorio incluye a 19.240 de estas empresas, acumulando el $98 \%$ del valor de las exportaciones. De ellas, 11.969 son manufactureras y acumulan el $73 \%$ del valor de la exportación de la población de exportadores regulares españoles en ese año. 
Para medir las aglomeraciones de los exportadores regulares, nuestra principal variable de interés, primero calculamos las relaciones de exportación EPD tomando como origen de exportación la provincia en la que la empresa tiene su sede social. El Gráfico 1 muestra la distribución de la aglomeración. Entre las más de 107.000 relaciones de exportación únicas que han creado las empresas exportadoras regulares entre 2001 y 2004 , en un $72 \%$ de los casos solo hay una empresa regular que realiza esa actividad de exportación en la provincia, mientras que en solo 1,6\% de las combinaciones provincia-producto-destino hay más de 11 empresas regulares ${ }^{4}$.

\section{GRÁFICO 1 \\ DISTRIBUCIÓN DE LA AGLOMERACIÓN EXPORTADORA DE LAS EMPRESAS REGULARES MANUFACTURERAS POR PROVINCIA-PRODUCTO-DESTINO}

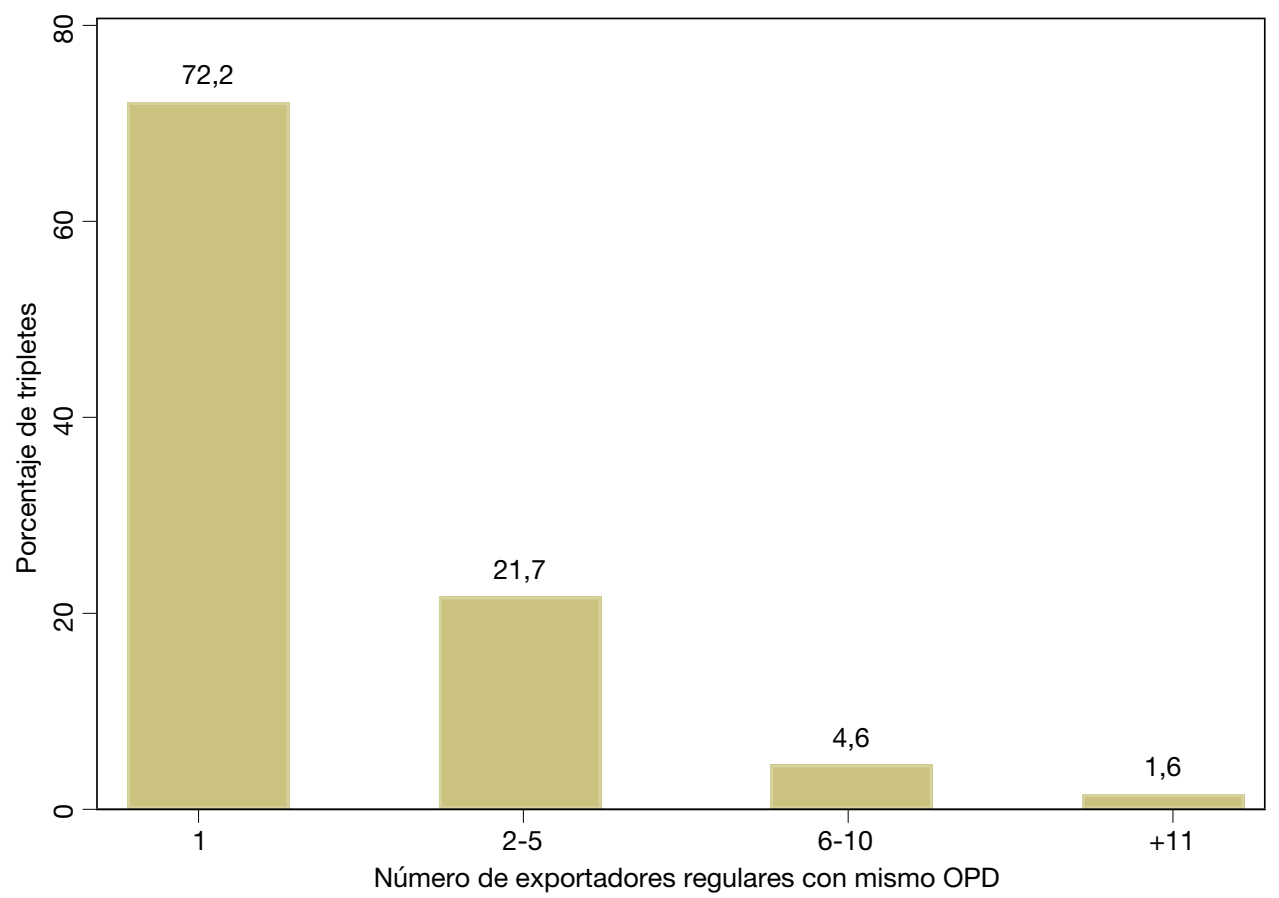

NOTA: Un OPD se refiere a la combinación provincia-producto-destino. Hay 203.268 EPD en total (107.696 si eliminamos las repetidas). Llamamos aglomeración OPD al número de EPD en un mismo origen geográfico. La provincia es la unidad de origen geográfico de la empresa basado en la localización de su establecimiento exportador.

FUENTE: Elaboración propia con datos de Cámaras-DIRMAN.

${ }^{4}$ También podemos comparar la distribución de aglomeraciones a nivel de producto-destino con las observadas en los casos en que las aglomeraciones se definen a nivel de producto o a nivel de destino (es decir, empresas ubicadas en la misma provincia que exportan el mismo producto o venden al mismo destino). El Gráfico A1 en el anexo muestra que las densidades de las aglomeraciones basadas en empresas exportadoras por producto-destino o por producto son menores a medida que aumenta el tamaño de la aglomeración. En el caso de las aglomeraciones por destino la densidad es más uniforme para distintos tamaños de aglomeración. 
Como primer paso antes de estimar el efecto de las aglomeraciones sobre la supervivencia de las nuevas relaciones de exportación, podemos analizar la distribución de empresas regulares que realizan la misma actividad exportadora donde hay nuevas empresas exportadoras. El Gráfico 2 muestra el porcentaje de empresas-producto-destinos observado para diferentes niveles de aglomeración. En general la densidad geográfica es escasa. El 38\% de las nuevas relaciones de exportación se inicia sin tener cerca ningún exportador regular realizando la misma actividad. Cuando hay exportadores regulares con la misma actividad exportadora, el rango varía mucho: mientras que un $16,4 \%$ de las nuevas relaciones comerciales solo tiene un exportador regular cercano, hay otro $14,3 \%$ que tienen más de 10 .

\section{GRÁFICO 2}

INCIDENCIA DE LAS AGLOMERACIONES DE EXPORTADORES REGULARES PARA LAS NUEVAS RELACIONES DE EXPORTACIÓN

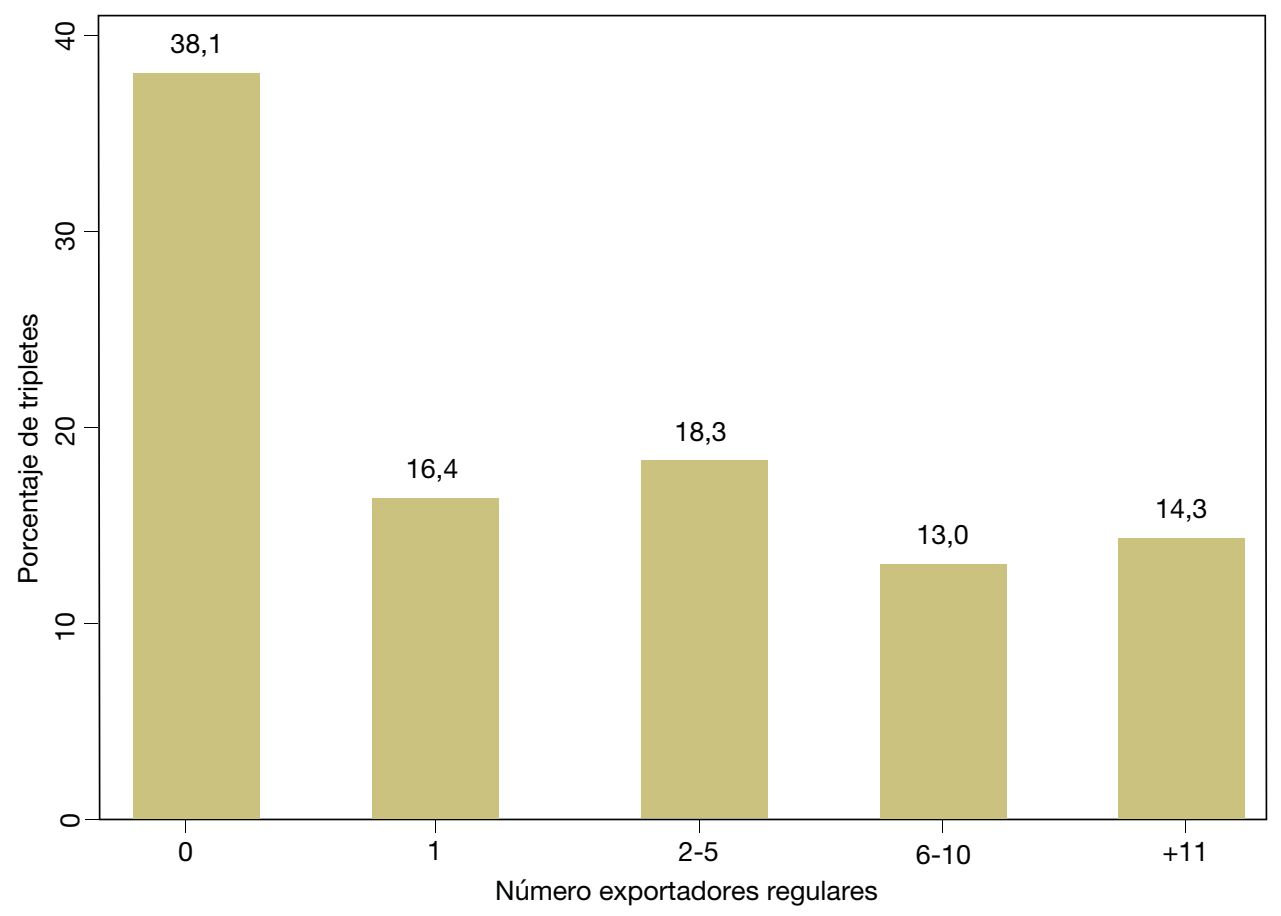

NOTA: Los exportadores nuevos crearon 2.740 EPD en 2004. Varios EPD pueden pertenecer a un mismo OPD o combinación provincia-producto-destino. Hay 2.535 OPD entre las nuevas empresas.

FUENTE: Elaboración propia con datos de Cámaras-DIRMAN 


\section{Especificación econométrica}

Nuestro análisis empírico está basado en la duración de las relaciones de exportación. Tenemos una ventana temporal que va de 2004 a 2018. Al analizar solo empresas que empiezan a exportar en 2004 no tenemos que preocuparnos de la historia exportadora anterior a 2004 (no hay censura por la izquierda). Sin embargo, la duración de una nueva actividad exportadora puede continuar después de 2018, por lo que no es posible determinar el valor de la duración completa (censura por la derecha). En este contexto, el modelo de regresión lineal genera inferencias sesgadas. En estas circunstancias, se recomienda estimar modelos de supervivencia, que permitan el análisis de la probabilidad de que un individuo cambie su estado, en nuestro caso, la probabilidad de que una empresa deje de exportar un producto a un destino. Para modelar el riesgo de transición hacia la desaparición del evento de interés, utilizamos una función de riesgo, $h(t)$, que se define como la probabilidad de que una nueva relación de exportación empresa-producto-destino termine en el año $t$, dado que ha sobrevivido al menos $t$ años.

Nuestra estrategia empírica se basa en una especificación de la tasa de riesgo condicionada a un conjunto de características observables $X$, es decir, $h(t, X)$. Como en otros trabajos empíricos que analizan la duración de las relaciones comerciales (Besedes y Prusa, 2006; Nitsh, 2009; Hess y Persson, 2012; Stirbat et al., 2015), usamos el modelo de Cox (Cox, 1972). Sin embargo, para acomodar la naturaleza de nuestros datos (frecuencia anual), usamos una versión discreta desarrollada por Prentice y Gloecker (1978), formalizado como:

$$
h(t, X)=1-\exp \left\{-\exp \left(x(t)^{\prime} \beta+\gamma_{t}\right)\right\}
$$

donde $\gamma_{t}$ es la función de riesgo de referencia (ya sea paramétrica o no paramétrica), es decir, captura el riesgo común para todas las unidades de análisis, $x(t)$ es un conjunto de características que puede cambiar con el tiempo y $\beta$ es el vector de parámetros de interés 5 . Además, ampliamos nuestra ecuación de estimación considerando heterogeneidad no observada $(u)$, que nos permite controlar por factores idiosincrásicos a cada relación de exportación (Heckman y Singer, 1984). La especificación final, tras una simple transformación, es:

$$
\begin{aligned}
\log \{-\log (1 & \left.\left.-h_{\text {fiopd }}(t, X)\right)\right\}= \\
& =\delta N_{\text {opd }}+\beta_{1}^{\prime} X_{f}+\beta_{2}^{\prime} X_{o}+\beta_{3}^{\prime} X_{i}+\beta_{4}^{\prime} X_{p}+\beta_{5}^{\prime} X_{d}+\gamma_{t}+u
\end{aligned}
$$

\footnotetext{
${ }^{5}$ Según Hess y Persson (2012), este enfoque es adecuado para modelar las relaciones comerciales, ya que los datos disponibles sobre la duración del comercio tienen un carácter discreto, dado que la duración de las relaciones tiene frecuencia anual. Dada esta característica, es habitual observar un gran número de relaciones comerciales con la misma duración (tiempos de supervivencia empatados), situación en la que los modelos de duración a tiempo continuo no son apropiados y pueden dar lugar a estimaciones y errores estándar sesgados.
} 
donde $h_{\text {fiopd }}(t, X)$ es la función de riesgo condicional para la empresa $f$ en la industria manufacturera $(i)$ en la provincia de origen $o$, que exporta el producto $p$ al mercado de destino $d$. Esta función de riesgo depende del número de exportadores regulares ubicados en la misma provincia, realizando una actividad de exportación similar al menos cuatro años consecutivamente $\left(N_{o p d}\right)$. Por tanto, $\delta$ contabiliza el impacto del $S E$, medido a través de la aglomeración de empresas con experiencia, en la tasa de riesgo. A su vez, $\gamma_{t}$ es la función de riesgo común, que captura el patrón general de probabilidad de transición en el tiempo. Este último se puede obtener a través de una especificación semiparamétrica que consiste en incluir variables dicotómicas para cada año en el que hay actividad de exportación ${ }^{6}$.

Nuestro modelo también incluye datos sobre las características de la actividad exportadora de la empresa $X_{f}$, de industria $X_{i}$, de producto $X_{p}$, de provincia $X_{o}$ y de destino $X_{d}$, todos referidos al año de comienzo del episodio, para controlar posibles factores que podrían afectar al efecto de los $S E$. Además de incluir dummies provinciales y de industria, también incluimos el número de empresas localizadas en una provincia y que realizan la misma actividad manufacturera, para controlar por aglomeración industrial. Las características de la actividad exportadora de la empresa incluyen el valor exportado inicial, el tamaño de la cartera (combinación de otros productos y destinos) y la experiencia importadora. A nivel de producto exportado, incluimos una dummy que toma valor 1 cuando España tiene ventaja comparativa en los mercados de exportación (índice de Balassa mayor a 1,25). En el Cuadro A1 del anexo se muestra la definición, la fuente original, así como la media, desviación estándar, mínimo y máximo de cada variable explicativa.

\section{Resultados}

En primer lugar, examinamos la función de supervivencia no paramétrica (Kaplan y Meier, 1958) para diferentes tamaños de aglomeración. El Gráfico 3 muestra que la distribución del riesgo de interrupción en cada año es estadísticamente diferente para cada tamaño de aglomeración, siendo menor cuanto mayor es el tamaño de la aglomeración ${ }^{7}$. En todos los casos, se observa que la supervivencia de las relaciones de exportación es más difícil en los primeros años, para luego reducirse el riesgo con la duración del episodio exportador. Sin embargo, este gráfico muestra resultados interesantes. Por ejemplo, en el primer año más del $75 \%$ de las nuevas relaciones de exportación originadas en aglomeraciones de gran tamaño (más de 10 empresas) sobreviven frente al $40 \%$ de aquellas que ocurren donde no hay otra empresa regular exportadora cercana. Transcurridos tres años, cerca del $50 \%$ de las nuevas relaciones se mantiene allí donde hay una aglomeración de más de 10 exportadores regulares con la misma actividad exportadora, frente a menos del $20 \%$ donde no hay aglome-

\footnotetext{
${ }^{6}$ En este trabajo no hay repetición de episodios ya que solo conocemos la duración del primer episodio de exportación (el iniciado en 2004).

${ }^{7}$ Las diferencias en la distribución de supervivencia por tamaño de la aglomeración se han contrastado con el test de Wilcoxon, confirmando que son funciones estadísticamente diferentes.
} 


\section{GRÁFICO 3}

FUNCIÓN DE SUPERVIVENCIA KAPLAN-MEIER PARA DIFERENTES NIVELES DE AGLOMERACIÓN

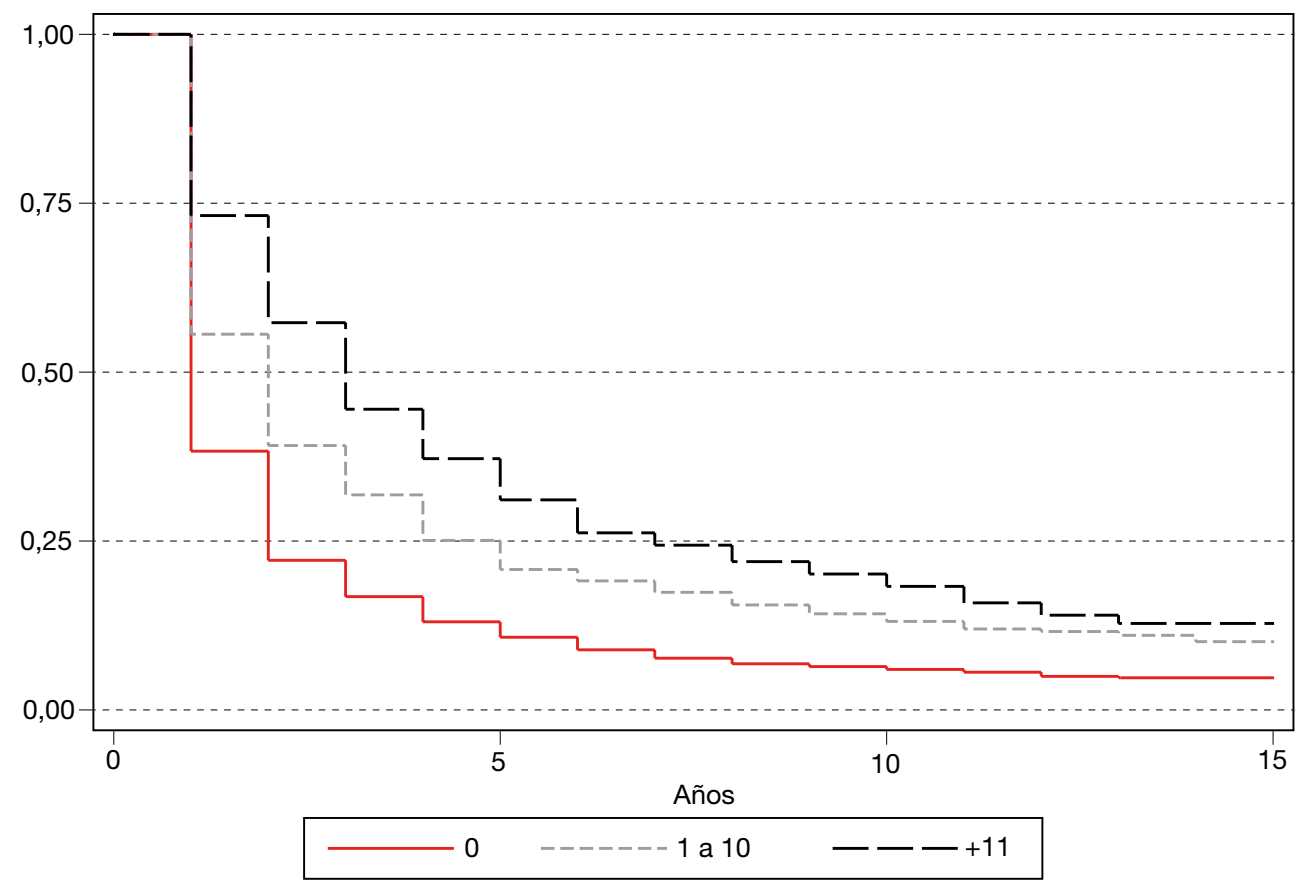

FUENTE: Elaboración propia.

ración. Parece, pues, que la presencia de un número elevado de empresas regulares compartiendo una misma actividad exportadora a nivel de producto-destino tiene un efecto positivo sobre la supervivencia de nuevas relaciones de exportación que se originan en esa provincia. Si este mecanismo funciona a lo largo del tiempo deberíamos esperar un circulo virtuoso entre aglomeración geográfica, supervivencia de nuevas empresas y consolidación de empresas regulares.

Sin embargo, el gráfico anterior no tiene en cuenta otros factores que pueden afectar a la duración de las relaciones de exportación. Usando el método de estimación propuesto, incluimos una batería de controles a nivel de empresa, industria, producto y destino, para evaluar con más precisión la importancia del tamaño de la aglomeración. Las estimaciones principales del trabajo se presentan en el Cuadro 5 utilizando el modelo econométrico propuesto cuando las variables de control se han incluido y el paso del tiempo se modeliza de forma semiparamétrica. En cada estimación se ha incluido al final el parámetro RHO, que se corresponde con la proporción de la varianza total que viene explicada por la heterogeneidad inobservable, y que es estadísticamente diferente de cero, indicando que no es posible descartar 
la existencia de características no observadas (por el investigador) de empresa que afectan a la duración de sus relaciones de exportación. Para facilitar la interpretación de los resultados, los coeficientes estimados se presentan en forma exponencial y deben ser interpretados de la siguiente manera: un valor por encima (debajo) de 1 indica un aumento (disminución) de la probabilidad de interrupción de la relación de exportación, lo que implica una menor (mayor) duración esperada ${ }^{8}$.

En la columna (1), el coeficiente asociado al número de exportadores regulares con el mismo origen-producto-destino es significativamente inferior a 1, luego la probabilidad de que una nueva empresa salga del mercado de exportación es menor a medida que aumenta el número de exportadores regulares con la misma actividad exportadora en esa provincia. En la columna (2) la variable $S E$ aparece dividida en 5 categorías según el número de exportadores regulares. La categoría omitida es aquella en la que una empresa empieza una actividad exportadora en una provincia en la que no hay ningún exportador regular cercano. Cuando otra empresa empieza a exportar y hay cerca una empresa regular realizando la misma actividad la probabilidad de salida del mercado de exportación se reduce un $41 \%(\beta=0,585 ; p$-valor $=0,00)$. A medida que el número de exportadores regulares aumenta hasta 10, la probabilidad de salida del mercado de exportación se reduce gradualmente. Sin embargo, cuando hacemos un test de igualdad de las diferencias de tamaño en el impacto sobre la probabilidad de salida no son estadísticamente significativas $(p$-valor $=0,22)$. Cuando el número de exportadores regulares es mayor de 10 , la probabilidad de salida se reduce notablemente hasta el $75 \%(\beta=0,248 ; p$-valor $=0,00)$ y es estadísticamente diferente de los coeficientes anteriores ( $p$-valor $=0,02)$.

Los resultados con respecto a las variables de control son los esperados. Los nuevos exportadores con un mayor grado de compromiso exportador, mayor tamaño de la cartera de productos y destinos, y que exportan a países de la zona euro, tienen menor probabilidad de dejar de exportar. Sin embargo, exportar a países de alto riesgo reduce la probabilidad de supervivencia exportadora. Además, tener una experiencia importadora previa en un producto-destino reduce drásticamente la duración de una nueva relación de exportación en ese producto-destino. Este resultado sugiere que la experiencia previa puede contribuir a reducir los costes hundidos específicos a esa actividad exportadora, facilitando la experimentación y, con ello, mayores tasas de entrada y salida en la actividad exportadora. También hay que destacar que la aglomeración de empresas manufactureras en una misma provincia (exporten o no) aumenta la probabilidad de interrupción de la actividad exportadora de empresas de la misma industria que empiezan a exportar; luego no parece que haya spillovers de tipo industrial. Por último, el coeficiente del resto de variables explicativas (tamaño del destino, distancia al destino, compartir una lengua común y pertenecer a la UE) tiene el signo esperado, pero no son estadísticamente significativas.

\footnotetext{
${ }^{8}$ En cada estimación se ha incluido un vector de dummies de año para cada año en el que un episodio ha existido. En todas las regresiones la dependencia de la duración resulta positiva, lo que implica que el riesgo de salida aumenta con el tiempo.
} 
En las columnas (3) y (4) volvemos a estimar la misma especificación con muestras de empresas diferentes. Primero seleccionamos solamente la relación de exportación más importante de la empresa. Pasar de no tener a tener un exportador regular con la misma actividad exportadora reduce la probabilidad de salida hasta un $73 \%(\beta=0,272 ; p$-valor $=0,00)$ y cuando hay más de 10 exportadores regulares la probabilidad cae al $95 \%(\beta=0,053 ; p$-valor $=0,00)$, siendo este impacto estadísticamente diferente del obtenido para número de empresas inferior a 10 ( $p$-valor $=0,02)$. A continuación, seleccionamos las relaciones de exportación de empresas que nunca han tenido ninguna experiencia importadora previa y empiezan exportando un único producto a un único destino. De nuevo se confirma que el impacto positivo de la presencia cercana de otros exportadores regulares.

Hasta el momento hemos demostrado que hay $S E$ positivos y que se originan a un nivel de provincia-producto-destino. Sin embargo, es posible que haya otros exportadores regulares fabricando el mismo producto y exportando a otros países o exportadores regulares vendiendo productos diferentes a un mismo país cuya actividad exportadora puede servir a los nuevos exportadores a reducir la probabilidad de interrupción de la actividad exportadora. Con el fin de comprobar el alcance de estas otras formas de $S E$, en el Cuadro 6 introducimos otras formas de medir la aglomeración: a nivel de provincia-producto, provincia-destino, y provincia 9 .

El Cuadro 6 utiliza como referencia las regresiones (1) y (2) del Cuadro 5, empleando primero una variable continua para medir las aglomeraciones (el logaritmo del número de empresas) y a continuación una variable categórica para tres tamaños de aglomeración: con cero empresas (la categoría omitida en la regresión), entre 1 y 10 empresas, y más de 10 empresas. Además, todas las regresiones incluyen las mismas variables de control, que han sido omitidas para economizar espacio.

Al introducir la aglomeración a nivel de provincia-producto (columna 1), una mayor aglomeración a nivel de provincia-producto-destino reduce la probabilidad de salida de la actividad exportadora $(\beta=0,60 ; p$-valor $=0,00)$. Sin embargo, una mayor aglomeración a nivel de provincia-producto aumenta la probabilidad de salida ( $\beta=1,18 ; p$-valor $=0,04)$. En la columna (2) observamos que la aglomeración generada por la presencia de exportadores regulares vendiendo a un mismo destino, aunque no produzcan el mismo producto, también reduce la probabilidad de salida $(\beta=0,74 ; p$-valor $=0,00)$. Además, aunque es de menor impacto, no podemos rechazar la hipótesis de que este coeficiente tenga el mismo valor que el obtenido para la aglomeración a nivel provincia-producto-destino $(\beta=0,69 ; p$-valor $=0,00)$. Los resultados se mantienen cuando incluimos las tres variables juntas (columna 3 ) y cuando incluimos al resto de exportadores regulares que operan en la provincia pero que no exportan el producto ni venden al país en el que lo hace la nueva empresa exportadora (columna 4).

\footnotetext{
9 En la construcción de estas variables las empresas se han clasificado de forma exclusiva y mutuamente excluyentes en cuatro categorías (OPD, OP, OD y OnoPnoD). La suma de las cuatro categorías es igual al número de exportadores regulares en la provincia $(\mathrm{O})$.
} 


\section{CUADRO 5}

ESTIMACIONES DEL MODELO ECONOMÉTRICO DE SUPERVIVENCIA

\begin{tabular}{|c|c|c|c|c|}
\hline & $\begin{array}{c}\text { (1) } \\
\text { Todos los } \\
\text { episodios }\end{array}$ & $\begin{array}{c}\text { (2) } \\
\text { Todos los } \\
\text { episodios }\end{array}$ & $\begin{array}{c}\text { (3) } \\
\text { Episodio } \\
\text { principal }\end{array}$ & \begin{tabular}{|c|} 
(4) \\
Episodios \\
únicos y sin \\
exper. Import.
\end{tabular} \\
\hline $\begin{array}{l}\text { spillover origen-producto-destino } \\
\text { (log número empresas) }\end{array}$ & $0,695 * * *$ & & & \\
\hline $\begin{array}{l}\text { spillover origen-producto-destino } \\
\text { (tamaño }=1 \text { empresa) }\end{array}$ & & $0,585^{* * *}$ & $0,272 * * *$ & $0,228 * *$ \\
\hline $\begin{array}{l}\text { spillover origen-producto-destino } \\
\text { (tamaño } 2 \text { a } 5 \text { empresas) }\end{array}$ & & $0,560 * * *$ & $0,174 * * *$ & $0,219 * *$ \\
\hline $\begin{array}{l}\text { spillover origen-producto-destino } \\
\text { (tamaño de } 6 \text { a } 10 \text { empresas) }\end{array}$ & & $0,403 * * *$ & $0,191 * * *$ & $0,248^{* *}$ \\
\hline $\begin{array}{l}\begin{array}{l}\text { spillover origen-producto-destino } \\
\text { (más de } 10 \text { empresas) }\end{array} \\
\end{array}$ & & $0,248 * * *$ & $0,053 * * *$ & $0,063 * * *$ \\
\hline log valor inicial exportación & $0,610 * * *$ & $0,619 * * *$ & $0,402 * * *$ & $0,350 * * *$ \\
\hline $\begin{array}{l}\text { dummy experiencia importadora origen- } \\
\text { producto-destino }\end{array}$ & $3,734 * * *$ & $3,644 * * *$ & $4,821 * * *$ & \\
\hline cartera 1 producto y $2-3$ destinos & $0,614 * * *$ & $0,605 * * *$ & 0,474 & \\
\hline cartera $2-3$ productos, 1 destino & $0,517 * * *$ & $0,521 * * *$ & $0,295^{* *}$ & \\
\hline cartera +3 productos, +3 destinos & $0,402 * * *$ & $0,407 * * *$ & 0,531 & \\
\hline$(\log )$ PIB & 0,983 & 0,990 & $0,699 * * *$ & $0,759 *$ \\
\hline$(\log )$ PIB per capita & 0,864 & 0,865 & 1,325 & 1,584 \\
\hline$(\log )$ distancia & 1,062 & 1,039 & 1,203 & 1,017 \\
\hline dummy lengua oficial espanol & 0,918 & 0,922 & 0,754 & 0,930 \\
\hline dummy pertenece a zona UE-no euro & 0,986 & 0,967 & 0,861 & 1,465 \\
\hline dummy pertenece a zona UE-euro & $0,630 * *$ & $0,614 * * *$ & $0,244 * *$ & $0,207 * *$ \\
\hline dummy riesgo-pais alto & $2,186 * *$ & $2,154 * *$ & $5,062 *$ & 2,363 \\
\hline $\begin{array}{l}\text { dummy Espana tiene ventaja comparativa } \\
\text { en ese producto }\end{array}$ & 1,191 & 1,214 & 0,726 & 0,582 \\
\hline (log) empresas manuf, en prov-industria & $1,090 *$ & $1,089 *$ & $1,235 *$ & $1,286^{*}$ \\
\hline RHO (heterogeneidad inobservada) & $0,520 * * *$ & $0,497 * * *$ & $0,889 * * *$ & $0,888 * * *$ \\
\hline $\begin{array}{l}\text { test }[\text { spill tamaño } 1]=[\text { tamaño } 2-5]= \\
\text { [tamaño 6-10] . [p-value] }\end{array}$ & & 0,22 & 0,61 & 0,98 \\
\hline $\begin{array}{l}\text { test [spill tamaño 6-10] }=[\text { tamaño }+10] \text {. } \\
\text { [p-value] }\end{array}$ & & 0,01 & 0,03 & 0,07 \\
\hline Número de observaciones & 11.566 & 11.566 & 4.371 & 2.151 \\
\hline Número de episodios & 2.716 & 2.716 & 1.222 & 741 \\
\hline Dummy Duracion & SI & SI & SI & SI \\
\hline Dummy Provincia & SI & SI & SI & SI \\
\hline Dummy Industria & SI & SI & SI & SI \\
\hline Log Likelihood & -4881 & -4871 & -1910 & $-983,3$ \\
\hline
\end{tabular}

NOTAS: Estimaciones de la función de riesgo obtenidas de un modelo proporcional de riesgo en tiempo discreto con constante y efectos aleatorios (utilizando el comando de Stata $x t c l o g l o g$ ). Coeficientes en forma exponencial: por encima (debajo) de 1 indica un aumento (disminución) de la probabilidad de interrupción de la relación de exportación. La significatividad de los coeficientes se indica con *** $1 \%$, ** 5\% y * $10 \%$.

FUENTE: Elaboración propia. 
Por último, en la columna (5) introducimos tres categorías de tamaño para cada tipo de aglomeración como variables explicativas. Para la aglomeración más específica a nivel provincia-producto-destino se repite la conclusión del Cuadro 5: la presencia de hasta 10 exportadores regulares con la misma actividad exportadora reduce la probabilidad de salida comparado con no haber ninguna empresa (53\%) y cuando

\section{CUADRO 6 \\ IMPACTO DE OTRAS FORMAS DE AGLOMERACIÓN SOBRE LA SUPERVIVENCIA}

\begin{tabular}{|c|c|c|c|c|c|c|c|}
\hline $\begin{array}{c}\text { Descripción del tipo } \\
\text { de aglomeración }\end{array}$ & Código & Construcción & (1) & (2) & (3) & (4) & (5) \\
\hline \multicolumn{8}{|l|}{ Variable continua } \\
\hline $\begin{array}{l}\text { Misma provincia - mis- } \\
\text { mo producto - mismo } \\
\text { destino }\end{array}$ & OPD & $\begin{array}{l}\text { (ln) número } \\
\text { empresas }\end{array}$ & $0,601 * * *$ & $0,690 * * *$ & $0,667 * * *$ & $0,667 * * *$ & \\
\hline $\begin{array}{l}\text { Misma provincia - } \\
\text { mismo producto - otros } \\
\text { destinos }\end{array}$ & OP & $\begin{array}{l}\text { (ln) número } \\
\text { empresas }\end{array}$ & $1,185^{* *} *$ & & 1,043 & 1,042 & \\
\hline $\begin{array}{l}\text { Misma provincia } \\
\text { - otros productos - } \\
\text { mismo destino }\end{array}$ & OD & $\begin{array}{l}\text { (ln) número } \\
\text { empresas }\end{array}$ & & $0,740 * * *$ & $0,751 * * *$ & $0,758 * *$ & \\
\hline $\begin{array}{l}\text { Misma provincia - } \\
\text { otros productos - otros } \\
\text { destinos }\end{array}$ & OnoPnoD & $\begin{array}{l}\text { (ln) número } \\
\text { empresas }\end{array}$ & & & & 0,956 & \\
\hline \multicolumn{8}{|l|}{ Variable categórica } \\
\hline $\begin{array}{l}\text { Misma provincia - mis- } \\
\text { mo producto - mismo } \\
\text { destino }\end{array}$ & OPD & $\begin{array}{l}\text { Núm. empresas } \\
{[1,10]}\end{array}$ & & & & & $0,466^{* * * *}$ \\
\hline $\begin{array}{l}\text { Misma provincia - mis- } \\
\text { mo producto - mismo } \\
\text { destino }\end{array}$ & OPD & $\begin{array}{l}\text { Núm. empresas } \\
{[+11]}\end{array}$ & & & & & $0,220 * * *$ \\
\hline $\begin{array}{l}\text { Misma provincia - } \\
\text { mismo producto - otros } \\
\text { destinos }\end{array}$ & OP & $\begin{array}{l}\text { Núm. empresas } \\
{[1,10]}\end{array}$ & & & & & 1,197 \\
\hline $\begin{array}{l}\text { Misma provincia - } \\
\text { mismo producto - otros } \\
\text { destinos }\end{array}$ & $\mathrm{OP}$ & $\begin{array}{l}\text { Núm. empresas } \\
{[+11]}\end{array}$ & & & & & $1,498^{*}$ \\
\hline $\begin{array}{l}\text { Misma provincia } \\
\text { - otros productos - } \\
\text { mismo destino }\end{array}$ & OD & $\begin{array}{l}\text { Núm. empresas } \\
{[11,40]}\end{array}$ & & & & & $0,625^{*}$ \\
\hline $\begin{array}{l}\text { Misma provincia } \\
\text { - otros productos - } \\
\text { mismo destino }\end{array}$ & OD & $\begin{array}{l}\text { Núm. empresas } \\
{[+41]}\end{array}$ & & & & & 0,750 \\
\hline Núm. de observaciones & & & 11.566 & 11.094 & 11.094 & 11.078 & 11.566 \\
\hline Log Likelihood & & & -4878 & -4588 & -4587 & -4576 & -4872 \\
\hline
\end{tabular}

NOTAS: Se han incluido todas las variables de control que aparecen en el Cuadro 5. Estimaciones xtcloglog. Coeficientes en forma exponencial. La significatividad de los coeficientes se indica con $* * * 1 \%$, ** 5\% y * $10 \%$. La suma de las empresas de las aglomeraciones OPD, OP, OD y OnoPnoD es igual al número total de empresas en la provincia $(\mathrm{O})$. Como no hay ninguna nueva empresa exportadora que empiece exportando a un país al que no exporte ninguna empresa regular anteriormente, la categoría omitida no puede ser cero; por ellos se ha construido la nueva categoría omitida para OD es $[0,10]$.

FUENTE: Elaboración propia. 
el número es mayor de 10 la reducción es mucho mayor (88\%). La aglomeración de exportadores regulares exportando un mismo producto aumenta el riesgo de interrupción de las nuevas relaciones de exportación. Finalmente, la aglomeración de exportadores regulares vendiendo al mismo destino cualquier producto reduce la probabilidad de salida. Sin embargo, la relación no es lineal ya que a medida que aumenta el tamaño de este tipo de aglomeración no se observa una mayor reducción en la probabilidad de salida.

\section{Conclusiones}

Utilizando datos de empresas manufactureras españolas en 2004, este trabajo encuentra un impacto positivo de las aglomeraciones o redes locales de empresas exportadoras regulares sobre la probabilidad de sobrevivir de las relaciones de exportación que emprenden las nuevas empresas exportadoras. En concreto, cuando el número de exportadores regulares que exporta el mismo producto al mismo país es superior a 10 la probabilidad de que finalice la nueva relación de exportación se reduce un $75 \%$. El impacto sobre la probabilidad de finalización de un episodio exportador obtenido para las empresas exportadoras regulares a nivel de producto-destino es mayor que el que se obtiene a nivel de provincia-producto o provincia-destino.

Una posible lectura de política económica es que una estrategia aparentemente viable para la política de promoción de exportaciones es potenciar el contacto entre empresas cercanas que desarrollan una misma actividad exportadora. Además, dado que esta externalidad es muy específica, justifica que este tipo de actividades de promoción de las exportaciones se deben diseñar a nivel muy específico (para combinaciones concretas de producto y destino) para que sean eficaces.

En relación con los programas de iniciación a la exportación cabría pensar en reorientar las actuaciones enfocando los esfuerzos de iniciación en combinaciones producto y país específicas para cada territorio en función del tejido empresarial que ya está exportando. Igualmente sería oportuno crear una base exportadora con suficiente número de empresas que permita favorecer la permanencia de las nuevas relaciones comerciales con el exterior. Este tipo de actuaciones podrían aumentar la eficiencia de los recursos destinados a la iniciación en la exportación reduciendo la pérdida de costes hundidos en los que la empresa incurre al iniciarse en su actividad exterior que no permanece en el tiempo. 


\section{Referencias bibliográficas}

Ahn J., Khandelwal, A., \& Wei, Sh. (2011). The role of intermediaries in facilitating trade. Journal of International Economics, 84(1), 73-85. https://doi.org/10.1016/j.jinteco.2010.12.003

Barrios, S., Gorg, H., \& Strobl, E. (2003). Explaining firm's export behaviour: R\&D, spillovers and the destination market. Oxford Bulletin of Economics and Statistics, 65(4), 475-496. https://doi.org/10.1111/1468-0084.t01-1-00058

Besedes, T., \& Prusa, T. (2006). Ins, outs and the duration of trade. Canadian Journal of Economics, 39(1), 266-295. https://doi.org/10.1111/j.0008-4085.2006.00347.x

Cassey, A. J., \& Schmeiser, K. N. (2013). The agglomeration of exporters by destination. Annals of Regional Science, 51(2), 495-513. https://doi.org/ 10.1007/s00168-012-0538-9

Choquette, E. (2019). Import-based market experience and firm's exit from export markets. Journal of International Business Studies, 50, 423-449. https://doi.org/ 10.1057/s41267-018-0193-1

Cox, D. R. (1972). Regression models and life-tables. Journal of the Royal Statistical Society, 34(2), 187-220. https://doi.org/10.1111/j.2517-6161.1972.tb00899.x

De Lucio, J., Mínguez, Minondo, A., \& Requena F. (2020). New exporters benefit more from information spillovers. Applied Economics Letters, 27(19), 1587-1591. https://doi.org/10.1080/13504851.2019.1698709

Duan J., Das, K., Meriluoto, L, \& Reed W. R. (2020). Estimating the effect of spillovers on exports: a meta-analysis. Review of World Economics, 156(2), 219-249.

https://doi.org/10.1007/s10290-020-00377-z

Esteve-Pérez, S., Pallardo-López, V., \& Requena-Silvente, F. (2013). The duration of firm-destination export relationships: Evidence from Spain, 1997-2006. Economic Inquiry, 51(1), 159-180. https://doi.org/10.1111/j.1465-7295.2012.00460.x

Esteve-Pérez, S, de Lucio, J., Mínguez, R., Minondo, A., \& Requena, F. (2017). La supervivencia exportadora. Un análisis a nivel de empresa, producto y destino. Cuadernos de Información Económica, 258, mayo/junio, 15-34.

Kaplan, E. L., \& Meier, P. (1958). Nonparametric estimation from incomplete observations. Journal of the American statistical association, 53(282), 457-481. https://doi.org/10.1080/01621459.1958.10501452

Heckman, J. J., \& Singer, B. (1984). A method for minimizing the impact of distributional assumptions in econometric models for duration data. Econometrica, 52(2), 271-320. https://doi.org/10.2307/1911491

Hess, W., \& Persson, M. (2012).The duration of trade revisited. Empirical Economics, 43(3), 1083-1107. https://doi.org/10.1007/s00181-011-0518-4

Krautheim, S. (2012). Heterogeneous firms, exporter networks and the effect of distance on international trade. Journal of International Economics, 87(1), 27-35. https://doi.org/10.1016/j.jinteco.2011.11.004

Mañez, J. A., Rochina, M. E., \& Sanchis, J. A. (2004). The decision to export: a panel data analysis for Spanish manufacturing. Applied Economics Letters, 11(11), 669-673. https://doi.org/10.1080/1350485042000236601

Muñoz-Sepúlveda, J., \& Rodríguez, D. (2015). Geographical and industrial spillovers in entry decisions across export markets. Applied Economics, 47(39), 4168-4183.

https://doi.org/10.1080/00036846.2015.1026582 
Nitsh, V. (2009). Die another day: Duration in German import trade. Review of World Economics, 145, 133-154. https://doi.org/10.1007/s10290-009-0008-3

Prentice, R. L., \& Gloeckler, L. A. (1978). Regression analysis of grouped survival data with application to breast cancer data. Biometrics, 34(1), 57-67. https://doi.org/10.2307/2529588

Rauch, J. E., \& Watson, J. (2003). Starting small in an unfamiliar environment. International Journal of Industrial Organization, 27(7), 1021-1042. https://doi.org/10.1016/S0167-7187(03)00018-3

Requena-Silvente, F., \& Castillo-Giménez, J. (2007). Information spillovers and the choice of export destination: A multinomial logit analysis of Spanish young SMEs. Small Business Economics, 28(1), 69-86. https://doi.org/10.1007/s11187-005-7324-3

Stirbat, L., Record, R., \& Nghardsaysone, K. (2015). The experience of survival: Determinants of export survival in Lao PDR. World Development, 76, 82-94. https://doi.org/10.1016/j.worlddev.2015.06.007 


\section{ANEXO \\ GRÁFICO A1 \\ COMPARACIÓN DE LA AGLOMERACIÓN SEGÚN COMPOSICIÓN DE LA RELACIÓN DE EXPORTACIÓN}
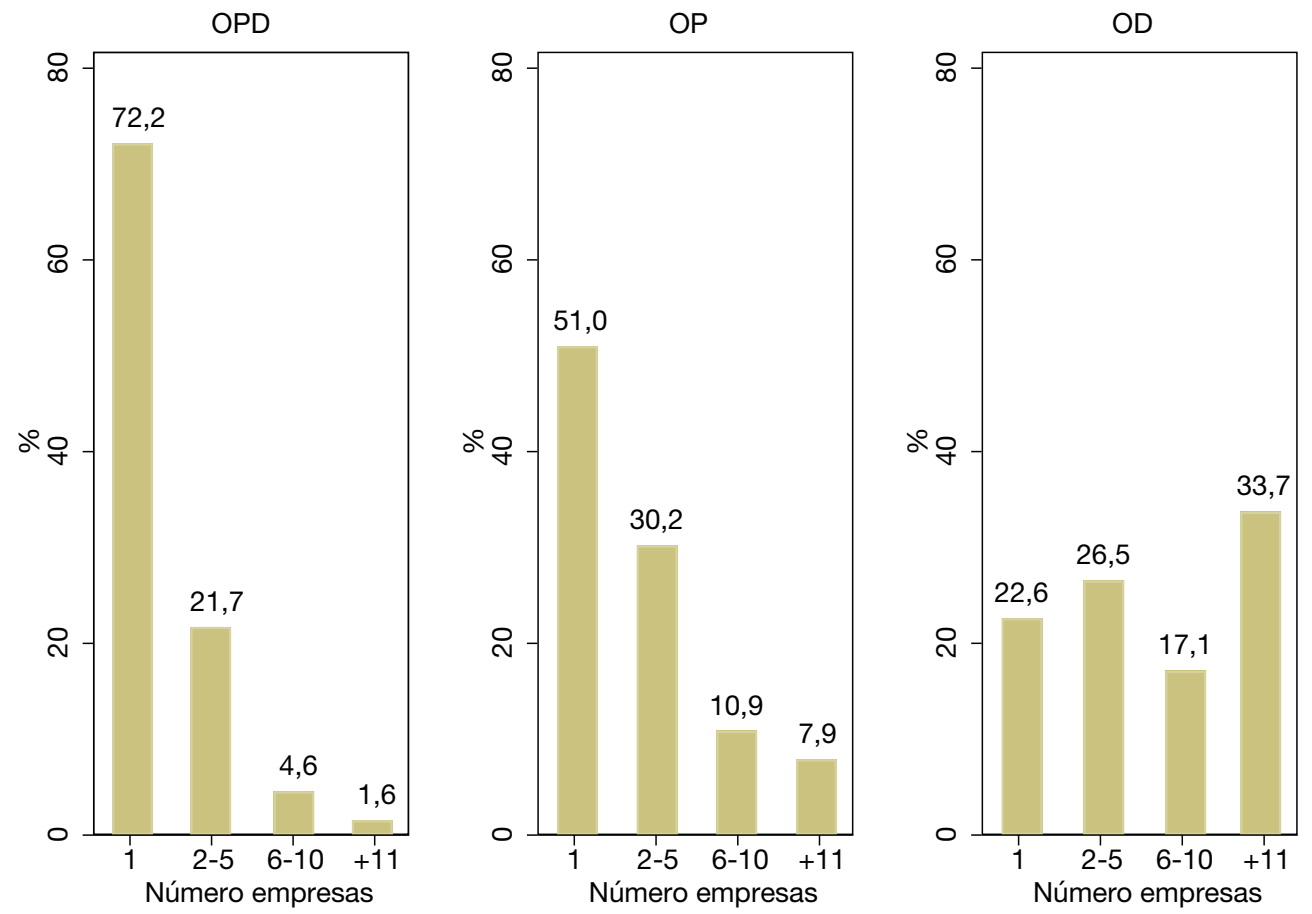

NOTA: Cada aglomeración se define según la definición de actividad exportadora. «Mismo origen-producto-destino» (OPD) se refiere a la aglomeración definida por el número de empresas en la misma provincia que exporta el mismo producto al mismo destino. Del mismo modo, «Mismo origen-producto» (OP) y «Mismo origen-destino» (OD) se refiere a la medida basada en el número de empresas, ubicadas en la misma provincia, que exportan el mismo producto o al mismo destino.

FUENTE: Elaboración propia con datos de Cámaras-DIRMAN. 
CUADRO A1

DESCRIPCIÓN DE LAS VARIABLES EXPLICATIVAS

\begin{tabular}{|c|c|c|c|c|c|c|}
\hline $\begin{array}{c}\text { Tipo } \\
\text { variable }\end{array}$ & $\begin{array}{l}\text { Nombre de } \\
\text { la variable }\end{array}$ & $\begin{array}{c}\text { Fuente } \\
\text { estadística }\end{array}$ & Media & Des. est. & Mínimo & Máximo \\
\hline \multirow[t]{4}{*}{ Spillovers } & $\begin{array}{l}\text { Spillover provincia-pro- } \\
\text { ducto-destino }\end{array}$ & AEAT-Aduanas & 8,85 & 21,60 & 0 & 172 \\
\hline & $\begin{array}{l}\text { Spillover provincia-pro- } \\
\text { ducto }\end{array}$ & AEAT-Aduanas & 37,56 & 67,08 & 0 & 324 \\
\hline & $\begin{array}{l}\text { Spillover provincia-des- } \\
\text { tino }\end{array}$ & AEAT-Aduanas & 395,87 & 551,49 & 0 & 2147 \\
\hline & Spillover provincia & AEAT-Aduanas & $1.240,68$ & $1.201,88$ & 12 & 3.141 \\
\hline \multirow[t]{6}{*}{ Empresa } & $\begin{array}{l}\text { Valor inicial de exporta- } \\
\text { ción (miles euros) }\end{array}$ & AEAT-Aduanas & 267,76 & $1.319,78$ & 6 & 50.627 \\
\hline & Experiencia importadora & AEAT-Aduanas & 0,03 & 0,18 & 0 & 1 \\
\hline & $\begin{array}{l}\text { Cartera con } \mathrm{N} \text { pto[=1] } \\
\text { Ndes }[=1]\end{array}$ & AEAT-Aduanas & 0,30 & 0,46 & 0 & 1 \\
\hline & $\begin{array}{l}\text { Cartera con N pto[=1] } \\
\text { Ndes }[=2-3]\end{array}$ & AEAT-Aduanas & 0,20 & 0,40 & 0 & 1 \\
\hline & $\begin{array}{l}\text { Cartera con N pto[=2-3] } \\
\text { Ndes }[=1]\end{array}$ & AEAT-Aduanas & 0,11 & 0,31 & 0 & 1 \\
\hline & $\begin{array}{l}\text { Cartera con } \mathrm{N} \text { pto [+3] } \\
\text { Ndes [+3] }\end{array}$ & AEAT-Aduanas & 0,40 & 0,49 & 0 & 1 \\
\hline \multirow[t]{7}{*}{ Destino } & PIB (miles mill \$) & WDI & $1.532,08$ & $2.857,80$ & 1 & 12.275 \\
\hline & $\begin{array}{l}\text { PIB per cápita (\$ cons- } \\
\text { tante 2010) }\end{array}$ & WDI & $29.058,43$ & $16.737,83$ & 703 & 116.418 \\
\hline & $\begin{array}{l}\text { Distancia geodésica } \\
\text { origen-destino }(\mathrm{kms})\end{array}$ & CEPII & $3.517,44$ & $3.492,68$ & 680 & 19.517 \\
\hline & $\begin{array}{l}\text { Destino es miembro de } \\
\text { la UE-noEuro }\end{array}$ & CEPII & 0,13 & 0,33 & 0 & 1 \\
\hline & $\begin{array}{l}\text { Destino es miembro de } \\
\text { la zona Euro }\end{array}$ & CEPII & 0,41 & 0,49 & 0 & 1 \\
\hline & $\begin{array}{l}\text { Destino con español } \\
\text { como lengua oficial }\end{array}$ & CEPII & 0,10 & 0,30 & 0 & 1 \\
\hline & $\begin{array}{l}\text { Destino con índice ries- } \\
\text { go país alto }\end{array}$ & OCDE & 0,05 & 0,21 & 0 & 1 \\
\hline Producto & $\begin{array}{l}\text { Producto con índice } \\
\text { ventaja comparativa } \\
\text { (Balassa) alto }\end{array}$ & BACI & 1,86 & 2,67 & 0 & 29 \\
\hline Provincia & $\begin{array}{l}\text { Número empresas } \\
\text { manufactureras provin- } \\
\text { cia-industria }\end{array}$ & SABI & 224,03 & 425,96 & 1 & 1.790 \\
\hline
\end{tabular}

